\title{
Numerical Solution of Sine-Gordon Equation with the Local Kriging Meshless Method
}

\author{
Pengfei Guo $\mathbb{D}^{1},{ }^{1}$ Ariunkhishig Boldbaatar, ${ }^{1}$ Dutao $Y i \mathbb{D}^{2},{ }^{2}$ and Pengxiang Dai ${ }^{2}$ \\ ${ }^{1}$ School of Mathematics and Statistics, Key Laboratory of Data Science and Intelligence Education, \\ Key Laboratory of Computer and Application of Hainan Province, Hainan Normal University, Haikou 571158, China \\ ${ }^{2}$ Key Laboratory for Wind and Bridge Engineering of Hunan Province, College of Civil Engineering, Hunan University, \\ Changsha 410082, China \\ Correspondence should be addressed to Dutao Yi; yidutao@hnu.edu.cn
}

Received 21 July 2020; Accepted 13 August 2020; Published 8 September 2020

Academic Editor: Yumin Cheng

Copyright $\odot 2020$ Pengfei Guo et al. This is an open access article distributed under the Creative Commons Attribution License, which permits unrestricted use, distribution, and reproduction in any medium, provided the original work is properly cited.

\begin{abstract}
This paper develops a local Kriging meshless solution to the nonlinear 2+1-dimensional sine-Gordon equation. The meshless shape function is constructed by Kriging interpolation method to have Kronecker delta function property for the two-dimensional field function, which leads to convenient implementation of imposing essential boundary conditions. Based on the local Petrov-Galerkin formulation and the center difference method for time discretization, a system of nonlinear discrete equations is obtained. The numerical examples are presented and the numerical solutions are found to be in good agreement with the results in the literature to validate the ability of the present meshless method to handle the $2+1$-dimensional sine-Gordon equation related problems.
\end{abstract}

\section{Introduction}

The nonlinear sine-Gordon equation (SGE), a type of hyperbolic partial differential equation, is often used to describe and simulate the physical phenomena in a variety of fields of engineering and science, such as nonlinear waves, propagation of fluxons and dislocation of metals [1-4]. Because sine-Gordon equation leads to different types of soliton solutions, SGE has been receiving an enormous amount of attention. Soliton solution travels without experiencing any deformation through the medium, even when it collides with another soliton. The solitons, identified in many wave and particle systems, are of importance in the theory of nonlinear differential equations.

As one of the crucial equations in nonlinear science, the sine-Gordon equation has been constantly investigated and solved numerically and analytically in recent years [4-8]. For the one-dimensional sine-Gordon equation, Wazwaz [9] adopted the tanh method to find the traveling wave solutions. Johnson et al. [10] provided three exact solutions to deal with the two-dimensional SGE. Zhong and Belić analytically established special two-soliton solution to solve the generalized SGE based on the Hirota bilinear method and self-similar method [11]. For more related research about exactly solving the sine-Gordon equations, the interested readers may refer to [12-15]. Despite the exact solutions or analytical analyses of the SGEs that can offer deeper understanding and insights of the associated physical phenomena, numerical methods are still indispensable in pursuit those unknown soliton solutions to the SGEs. Dehghan et al. used the collocation method based on the radial basis function to obtain the numerical solutions to the one-dimensional nonlinear sine-Gordon equation [16]. Besides, the difference approach [17], the boundary integral equation scheme [18], the homotopy-perturbation method [19], the modified Adomain decomposition method [20], and the multilevel augmentation method [21] have also been applied to solve the one-dimensional SGEs. For dealing with the higher dimensional problems, Argyris et al. [22] proposed a finite element algorithm to obtain soliton-type solutions. Djidjeli et al. [23] developed an explicit numerical approach to solve a damped sine-Gordon equation in two 
space variables. Sheng et al. presented a split cosine scheme [24], and Bratsos used the method of lines [25] for seeking the solitary solutions of the two-dimensional sine-Gordon equation.

During recent decades, meshless methods have also been developed for solving the nonlinear sine-Gordon equations, which include the mesh-free $k p$-Ritz method [4], the radius basis function (RBF) method $[7,16]$, the radial point interpolation method (RPIM) [8], the meshless local PetrovGalerkin (MLPG) method [26], and the well-posed moving least-squares (WP-MLS) approximation [27]. Meshless methods are a type of numerical schemes by which the problem domain is represented by a set of scattered nodes, and the mesh or discretization can thus be avoided. They have been usually used to effectively handle those physical and engineering problems or to solve the partial differential equations that might pose challenges of the traditional numerical approaches, such as the boundary element method [28] and finite element method [29]. A variety of meshless methods have been developed and then applied in scientific and engineering modelling based on different combinations of certain formulation schemes, such as the element-free Galerkin formulation [30], and the approximation/interpolation techniques, such as moving least-squares (MLS) $[31,32]$. Based on MLS, the element-free Galerkin method has been viewed as one of the predominant meshless methods. Cheng et al. proposed the improved moving least-squares (IMLS) approximation to develop the improved element-free Galerkin (IEFG) method [33-35] and the interpolating element-free Galerkin method [36-38]. By introducing the dimension splitting method into the reproducing kernel particle method, a new meshless method was developed to solve three-dimensional potential problems [39-43].

In this study, we present the local Kriging meshless method [44-47] to seek the numerical solutions of the $2+1$ dimensional nonlinear sine-Gordon equation. The present meshless approach is based on the Kriging interpolation technique and the local Petrov-Galerkin formulation, which leads to the shape functions having the Kronecker delta property and thus to a convenient implementation required for imposing the essential boundary conditions, like in the traditional finite element analysis. Combining with the Petrov-Galerkin formulation over the local subdomains, the meshless method is featured by avoiding background cells for the numerical integration of the global weak form. Based on the center difference scheme for making time discretization, we have a global system of nonlinear algebraic equations. Numerical tests are performed to validate the convergence and accuracy of the present meshless method for solving the $2+1$-dimensional sine-Gordon equation. The numerical results are found to agree well with the existing results reported in the published literature.

\section{The Local Kriging Meshless Formulation for the 2D Sine-Gordon Equation}

2.1. Governing Equation. In the present study, we seek to acquire a meshless approximation to the damped $2+1$-dimensional sine-Gordon equation [4],

$$
\begin{aligned}
\frac{\partial^{2} u(x, t)}{\partial t^{2}}+\beta \frac{\partial u(x, t)}{\partial t}= & \frac{\partial^{2} u(x, t)}{\partial x^{2}}+\frac{\partial^{2} u(x, t)}{\partial y^{2}} \\
& -\phi(x, y) \sin (u(x, t)),
\end{aligned}
$$

within the problem domain of $\Omega=\{(x, y) \mid a \leq x$ $\leq b, c \leq y \leq d\}$ for $t>0$. In equation (1), the parameter $\beta \geq 0$ is the damping factor for the dissipative term. When $\beta=0$, this equation will reduce to an undamped sine-Gordon equation. The functions $\phi(x, y)$ represent Josephson current density.

The boundary conditions linked to the sine-Gordon equation (equation (1)) enforce a zero normal gradient on the boundary $\Gamma$ of $\Omega$ as follows:

$$
\frac{\partial u(x, y, t)}{\partial n}=0, \quad(x, y) \in \Gamma .
$$

At time $t=0$, we assume the initial conditions to be in the form of

$$
\left\{\begin{array}{l}
u(x, y, 0)=\varphi_{1}(x, y), \\
\frac{\partial u(x, y, 0)}{\partial t}=\varphi_{2}(x, y)
\end{array}\right.
$$

where the functions, $\varphi_{1}(x, y)$ and $\varphi_{2}(x, y)$, are specified wave modes and velocity.

2.2. Kriging Interpolation. The problem domain $\Omega$ with the boundary $\Gamma$ can be represented by a set of scattered nodes, the $I^{\text {th }}$ of which is assumed to be located at $\mathbf{x}_{I}(I=1,2, \ldots, N)$, where $N$ is the total number of nodes. The field function, such as $u(x)$, is approximated/interpolated via the nodal values $u\left(\mathbf{x}_{I}\right)$. Through a weighted linear combination, the field value $u\left(\mathbf{x}_{0}\right)$ at point $\mathbf{x}_{0}$ can be evaluated approximately as

$$
\widehat{u}\left(\mathbf{x}_{0}\right)=\sum_{I=1}^{n} \lambda_{I} u\left(\mathbf{x}_{I}\right), \quad\left(\mathbf{x}_{I} \in \Omega_{s}\right),
$$

where $u\left(\mathbf{x}_{I}\right)$ stands for the nodal value at $\mathbf{x}_{I}(I=1,2, \ldots, n)$ and $\Omega_{s}\left(\Omega_{s} \subset \Omega\right)$ represents the support domain associated with the point at $\mathbf{x}_{0}$, which contains $n$ nodes that exert influence on the point at $\mathbf{x}_{0}$. The weight coefficient $\lambda_{I}$ will be determined according to the Kriging interpolation method and the Kriging-based shape function can thus be written by

$$
\widehat{u}\left(\mathbf{x}, \mathbf{x}_{I}\right)=\boldsymbol{\Psi}\left(\mathbf{x}, \mathbf{x}_{I}\right) \mathbf{u}_{I}=\left[\mathbf{p}(\mathbf{x})^{T} \mathbf{A}\left(\mathbf{x}_{I}\right)+\mathbf{r}(\mathbf{x})^{T} \mathbf{B}\left(\mathbf{x}_{I}\right)\right] \mathbf{u}_{I} .
$$

The matrices $\mathbf{A}\left(\mathbf{x}_{I}\right)$ and $\mathbf{B}\left(\mathbf{x}_{I}\right)$ are expressed, respectively, as

$$
\begin{aligned}
& \mathbf{A}\left(\mathbf{x}_{I}\right)=\left(\mathbf{P}^{T} \mathbf{R}^{-1} \mathbf{P}\right)^{-1} \mathbf{P}^{T} \mathbf{R}^{-1}, \\
& \mathbf{B}\left(\mathbf{x}_{I}\right)=\mathbf{R}^{-1}(\mathbf{I}-\mathbf{P A}),
\end{aligned}
$$

where $\mathbf{I}$ is the unit matrix of order $n$, and $\mathbf{p}(\mathbf{x})$ is a column vector that contains $m$ monomial basis functions, which are 
given, as an example, in case of two-dimensional linear and quadratic basis functions, as follows:

$$
\begin{aligned}
& \mathbf{p}^{T}(\mathbf{x})=\left\{\begin{array}{lll}
1 & x & y
\end{array}, \quad(m=3),\right. \\
& \mathbf{p}^{T}(\mathbf{x})=\left\{\begin{array}{llllll}
1 & x & y & x^{2} & x y & y^{2}
\end{array}\right\}, \quad(m=6) .
\end{aligned}
$$

In terms of the basis function at nodes in the support domain, the matrix $\mathbf{P}$ is then written as

$$
\mathbf{P}=\left[\begin{array}{ccc}
p_{1}\left(\mathbf{x}_{1}\right) & \cdots & p_{m}\left(\mathbf{x}_{1}\right) \\
\vdots & \ddots & \vdots \\
p_{1}\left(\mathbf{x}_{n}\right) & \cdots & p_{m}\left(\mathbf{x}_{n}\right)
\end{array}\right]
$$

The matrix $\mathbf{R}$ and vector $\mathbf{r}(\mathbf{x})$ are, respectively, expressed as

$$
\begin{aligned}
& \mathbf{R}=\left[\begin{array}{ccc}
\gamma\left(\mathbf{x}_{1}, \mathbf{x}_{1}\right) & \cdots & \gamma\left(\mathbf{x}_{1}, \mathbf{x}_{n}\right) \\
\vdots & \ddots & \vdots \\
\gamma\left(\mathbf{x}_{n}, \mathbf{x}_{1}\right) & \cdots & \gamma\left(\mathbf{x}_{n}, \mathbf{x}_{n}\right)
\end{array}\right], \\
& \mathbf{r}^{T}(\mathbf{x})=\left[\gamma\left(\mathbf{x}, \mathbf{x}_{1}\right) \gamma\left(\mathbf{x}, \mathbf{x}_{2}\right) \gamma\left(\mathbf{x}, \mathbf{x}_{n}\right)\right],
\end{aligned}
$$

each entry of which, $\gamma\left(\mathbf{x}_{I}, \mathbf{x}_{J}\right)$, is evaluated based on the semivariogram model that determines the relation of any pair of nodes at $\mathbf{x}_{I}$ and $\mathbf{x}_{J}$ as follows:

$$
\gamma\left(\mathbf{x}_{I}, \mathbf{x}_{J}\right)=\frac{1}{2} E\left[\left(Z_{b}\left(\mathbf{x}_{I}\right)-Z_{b}\left(\mathbf{x}_{J}\right)\right)^{2}\right]=\gamma(\mathbf{h}),
$$

where $E[\cdot]$ stands for an expected value of a random function. The semivariogram model in equation (11) is independent of the locations of the two nodes but only depends on their lag vector $\mathbf{h}$.

In this numerical investigation, we adopt the Gauss model to construct the shape functions via the Kriging interpolation scheme [48], which is given as

$$
\gamma(h)=c_{0}\left[1-\exp \left(-\frac{3 h^{2}}{a_{0}^{2}}\right)\right]\left(h \leq a_{0}\right) .
$$

In equation (12), $h$ is the Euclidean distance of any point at $\mathbf{x}$ and the node at $\mathbf{x}_{I}$, or the distance of any pair of nodes at $\mathbf{x}_{I}$ and $\mathbf{x}_{J}$. Thus, $h=\left\|\mathbf{x}-\mathbf{x}_{I}\right\|$ for equation (10) and $h=\| \mathbf{x}_{I}-$ $\mathbf{x}_{J} \|$ for equation (9). The two factors $a_{0}$ and $c_{0}$ are the sill and range that are empirically determined. In the present study, we set $c_{0}$ to 1 because its value has little influence on the shape functions, whereas $a_{0}$ is evaluated according to the influence domain size $\left(d_{s}\right)$ linked to nodes

$$
a_{0}=\alpha_{0} \cdot d_{s}, \quad\left(\alpha_{0} \geq 1\right),
$$

in which $\alpha_{0}$ is a specified factor [46]. In the local Kriging meshless method, the interpolation domain for any point at $\mathbf{x}$ is constructed in terms of the influence domain size of $d_{I}$ that is written as

$$
d_{I}=\beta_{0} \cdot d_{\mathrm{ave}},
$$

where $\beta_{0}$ is a dimensional coefficient and $d_{\text {ave }}$ represents the average spacing among nodes, which is determined by considering the balance of computational costs and accuracy $[49,50]$.

In the problem domain $\Omega$, we have the derivatives of the constructed shape functions with respect to $x_{1}$ and $x_{2}$ based on equation (5), and their first- and second-order partial derivatives are given as follows:

$$
\begin{gathered}
\Psi_{, i}\left(\mathbf{x}, \mathbf{x}_{I}\right)=\mathbf{p}_{, i}(\mathbf{x})^{T} \mathbf{A}\left(\mathbf{x}_{I}\right)+\mathbf{r}_{, i}(\mathbf{x})^{T} \mathbf{B}\left(\mathbf{x}_{I}\right), \\
\Psi_{, i j}\left(\mathbf{x}, \mathbf{x}_{I}\right)=\mathbf{p}_{, i j}(\mathbf{x})^{T} \mathbf{A}\left(\mathbf{x}_{I}\right)+\mathbf{r}_{, i j}(\mathbf{x})^{T} \mathbf{B}\left(\mathbf{x}_{I}\right),
\end{gathered}
$$

in which ()$_{, i}$ and ()$_{, i j}$ represent $\partial() / \partial x_{i}$ and $\partial^{2}() / \partial x_{i} \partial x_{j}$, respectively.

2.3. Local Weak Form of the Governing Equation. In the present study, we apply the local Petrov-Galerkin formulation to construct the weak form of the governing equation over the pre-established local subdomains $\left(\Omega_{q}\right)$ associated with the nodes in the global problem domain $\Omega$ (see Figure 1). The positive definite test functions $\widehat{W}_{I}$ that are assigned to each node need to be predefined within a test function region $\left(\Omega_{w}\right)$. Over one local subdomain $\Omega_{q I}$ centered at node $I$, the local weighted residual weak form of equation (1) is then expressed as

$$
\int_{\Omega_{q I}} \widehat{W}_{I}\left[\frac{\partial^{2} u}{\partial t^{2}}+\beta \frac{\partial u}{\partial t}-\frac{\partial^{2} u}{\partial x^{2}}-\frac{\partial^{2} u}{\partial y^{2}}+\phi(x, y) \sin (u)\right] \mathrm{d} \Omega=0,
$$

where we specify a cubic spine function [44] as the test function $\widehat{W}_{I}$ in this study, which is defined as

$$
\widehat{W}_{I}=\widehat{W}\left(\mathbf{x}, \mathbf{x}_{I}\right)= \begin{cases}\frac{2}{3}-4 \bar{r}_{I}^{2}+4 \bar{r}_{I}^{3}, & \bar{r}_{I} \leq 0.5, \\ \frac{4}{3}-4 \bar{r}_{I}+4 \bar{r}_{I}^{2}-\frac{4 \bar{r}_{I}^{3}}{3}, & 0.5<\bar{r}_{I} \leq 1, \\ 0, & \bar{r}_{I}>1,\end{cases}
$$

in which $\overline{r_{I}}=\left(\left\|\mathbf{x}-\mathbf{x}_{I}\right\|\right) / r_{w}$.

By decomposing the subdomain boundary $\Gamma_{q}$ into three sub-boundaries and applying the Gauss divergence theorem, equation (16) is re-expressed as

$$
\begin{aligned}
& \int_{\Omega_{q I}} \widehat{W}_{I}\left(\frac{\partial^{2} u}{\partial t^{2}}+\beta \frac{\partial u}{\partial t}\right) \mathrm{d} \Omega-\int_{\Gamma_{q I i}+\Gamma_{q I u}} \widehat{W}_{I}\left(n_{x} \frac{\partial u}{\partial x}+n_{y} \frac{\partial u}{\partial y}\right) \mathrm{d} \Gamma \\
& \quad+\int_{\Omega_{q I}}\left(\widehat{W}_{I, x} \frac{\partial u}{\partial x}+\widehat{W}_{I, y} \frac{\partial u}{\partial y}\right) \mathrm{d} \Omega \\
& =\int_{\Gamma_{q I t}} \widehat{W}_{I}\left(n_{x} \frac{\partial u}{\partial x}+n_{y} \frac{\partial u}{\partial y}\right) \mathrm{d} \Gamma-\int_{\Omega_{q I}} \widehat{W}_{I}[\phi(x, y) \sin (u)] \mathrm{d} \Omega,
\end{aligned}
$$

in which $n_{x}$ and $n_{y}$ stand for the outward normal direction cosines of the boundary ( $\left.\Gamma_{q I}\right)$ of the local subdomain $\Omega_{q I}$ 


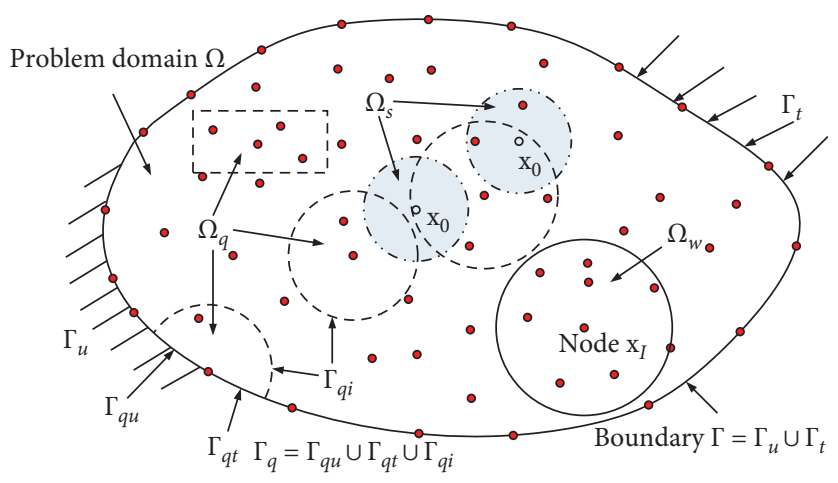

Figure 1: Local quadrature, support, and test function domains.

and the boundary contains three disjoint parts $\left(\Gamma_{q I}=\Gamma_{q I i} \cup \Gamma_{q I u} \cup \Gamma_{q I t}\right)$.

According to the Kriging interpolation method and the constructed shape function (equation (5)) presented in the previous section, the field function $u(\mathbf{x})$ to be determined can be approximated in terms of nodal values at the $n$ nodes that are included in the support domain associated with the point at $\mathbf{x}$ as follows:

$$
u^{h}(\mathbf{x}, t)=\sum_{J=1}^{n} \psi_{J}(\mathbf{x}) u\left(\mathbf{x}_{J}, t\right)=\sum_{J=1}^{n} \psi_{J} u_{J} .
$$

The time and space derivatives of the filed function are expressed in the following form of

$$
\begin{aligned}
& \frac{\partial u(\mathbf{x}, t)}{\partial t}=\left[\sum_{J=1}^{n} \psi_{J}(\mathbf{x}) \frac{\partial u\left(\mathbf{x}_{J}, t\right)}{\partial t}\right]=\Psi(\mathbf{x}) \dot{\mathbf{U}}(t) \\
& \frac{\partial^{2} u(\mathbf{x}, t)}{\partial t^{2}}=\left[\sum_{J=1}^{n} \psi_{J}(\mathbf{x}) \frac{\partial u^{2}\left(\mathbf{x}_{J}, t\right)}{\partial t^{2}}\right]=\Psi(\mathbf{x}) \ddot{\mathbf{U}}(t) \\
& \frac{\partial u(\mathbf{x}, t)}{\partial x_{i}}=\left[\sum_{J=1}^{n} \frac{\partial \psi_{J}(\mathbf{x})}{\partial x_{i}} u\left(\mathbf{x}_{J}, t\right)\right]=\Psi_{i}(\mathbf{x}) \mathbf{U}(t),
\end{aligned}
$$

where

$$
\begin{aligned}
& \Psi(\mathbf{x})=\left\{\begin{array}{llll}
\psi_{1}(\mathbf{x}) & \psi_{2}(\mathbf{x}) & \ldots & \psi_{n}(\mathbf{x})
\end{array}\right\}, \\
& \mathbf{U}(t)=\left\{\begin{array}{llll}
u_{1}(t) & u_{2}(t) & \ldots & u_{n}(t)
\end{array}\right\}^{T} .
\end{aligned}
$$

Substituting the approximate field function $u^{h}(\mathbf{x})$ in equation (19) for $u(\mathbf{x})$ in the local weak form of the governing equation (equation (18)), we obtain the nodal system equation for the $I$ th node in the matrix form:

$$
\mathbf{C}_{I} \ddot{\mathbf{U}}+\beta \mathbf{C}_{I} \dot{\mathbf{U}}+\mathbf{K}_{I} \mathbf{U}=\mathbf{F}_{I}(\mathbf{U}),
$$

in which

$$
\begin{aligned}
\mathbf{C}_{I} & =\int \widehat{W}_{I} \boldsymbol{\Psi}(\mathbf{x}) \mathrm{d} \Omega, \\
\mathbf{K}_{I} & =\int_{\Omega_{q I}}\left[\widehat{W}_{I, x} \Psi_{, x}(\mathbf{x})+\widehat{W}_{I, y} \Psi_{, y}(\mathbf{x})\right] \mathrm{d} \Omega-\int_{\Gamma q I i+\Gamma q I u} \widehat{W}_{I}\left[n_{x} \Psi_{, x}(\mathbf{x})+n_{y} \Psi_{, y}(\mathbf{x})\right] \mathrm{d} \Gamma, \\
\mathbf{F}_{I}(\mathbf{U}) & =-\int_{\Omega_{q I}} \widehat{W}_{I}[\phi(x, y) \sin (u)] \mathrm{d} \Omega+\int_{\Gamma_{q I t}} \widehat{W}_{I}\left(n_{x} \frac{\partial u}{\partial x}+n_{y} \frac{\partial u}{\partial y}\right) \mathrm{d} \Gamma .
\end{aligned}
$$

In order to construct the global system equation, the assembling process similar to the finite difference method has been used for all $N$ nodes scattered in the problem domain $\Omega$ and it leads to

$$
\mathbf{C} \ddot{\mathbf{U}}+\beta \mathbf{C U}+\mathbf{K U}=\mathbf{F}(\mathbf{U}) .
$$

Apparently, equation (26) is a system of nonlinear algebraic equations, and an iterative technique is then applied to cope with the nonlinearity. In equation (25), the first term on the right-hand side needs to be evaluated according to the latest available approximation of the field function $u$. 
2.4. The Time Discretization and Iterative Procedure. In the present study, the time derivatives of the global system equations (equation (25)) is handled by the center difference technique for making time discretization, and the global system equation (equation (25)) is transformed into

$$
\begin{aligned}
\mathbf{C} & \frac{\mathbf{U}^{(k+2)}-2 \mathbf{U}^{(k+1)}+\mathbf{U}^{(k)}}{\Delta t^{2}}+\beta \mathbf{C} \frac{\mathbf{U}^{(k+1)}-\mathbf{U}^{(k)}}{\Delta t} \\
& +\mathbf{K} \frac{\mathbf{U}^{(k+1)}+\mathbf{U}^{(k)}}{2}=\mathbf{F} .
\end{aligned}
$$

The above equation can be rewritten as

$$
\begin{aligned}
2 \mathbf{C} \mathbf{U}^{(k+2)}= & \left(4 \mathbf{C}-2 \beta \mathbf{C} \Delta t-\mathbf{K} \Delta t^{2}\right) \mathbf{U}^{(k+1)} \\
& +\left(-2 \mathbf{C}+2 \beta \mathbf{C} \Delta t-\mathbf{K} \Delta t^{2}\right) \mathbf{U}^{(k)}+2 \Delta t^{2} \mathbf{F} .
\end{aligned}
$$

When $t=0, \mathbf{U}^{(0)}$ is determined according to the specified initial condition (equation (3)), that is, $\mathbf{U}^{(0)}=\varphi_{1}(x, y)$, and for the next time level, $\mathbf{U}^{(1)}$ can be obtained as $\mathbf{U}^{(1)}=\mathbf{U}^{(0)}+2 \Delta t \varphi_{2}(x, y)$. For the purpose of handling the nonlinearity of equation (28) using a predictor-corrector technique $[4,26]$, at a time level of $t=k+1$, we need the field function value $\widetilde{\mathbf{U}}=\mathbf{U}^{(k)}$ at the last time level $(t=k)$ to calculate the term $2 \Delta t^{2} \mathbf{F}$ in the equation, and the system of equations is then solved linearly with $\mathbf{U}^{(k+2)}=\mathbf{U}^{(k+2), 0}$. Afterwards, we recalculate $\widetilde{\mathbf{U}}=(1 / 3)\left[\mathbf{U}^{(k+2), 0}+\mathbf{U}^{(k+1)}+\right.$ $\mathbf{U}^{(k)}$ ]. Equation (28) can be solved with the latest available $\widetilde{\mathbf{U}}$ to obtain the field function values $\mathbf{U}^{(k+2), 1}$ at the time level of $(k+2)$. Performing repeatedly this iterative procedure and setting $\tilde{\mathbf{U}}=(1 / 3)\left[\mathbf{U}^{(k+2), l}+\mathbf{U}^{(k+1)}+\mathbf{U}^{(k)}\right]$, we then achieve the nonlinear solutions to equation (28) when $t=k+2$ if the solutions can converge to a prescribed number $\varepsilon$. In the present study, the condition for terminating the iteration is given as follows:

$$
\left\|\mathbf{U}^{(k+2), l}-\mathbf{U}^{(k+2), l-1}\right\| \leq \varepsilon .
$$

We can set $\mathbf{U}^{(k+2)}=\mathbf{U}^{(k+2), l}$ and proceed to the next time level, in case the above condition is met.

By conducting the above iterative procedure to solve the equation (equation (28)) till the desired time level, we achieve the numerical solutions to the 2+1-dimensional nonlinear sine-Gordon equation.

\section{Numerical Examples}

In this section, we apply the local Kriging meshless method to several numerical examples regarding two-dimensional line and ring solitons.

\subsection{Example 1: Superposition of Two Orthogonal Line Solitons} (Undamped and Damped SG Equation) $[4,26,51]$. In the case of an undamped 2D sine-Gordon equation $(\beta=0)$ with $\phi(x, y)=1$, we obtain the superposition of two orthogonal line solitons under the given initial conditions,

$$
\left\{\begin{array}{l}
u(x, y, 0)=4 \tan ^{-1} \exp (x)+4 \tan ^{-1} \exp (y) \\
\frac{\partial u(x, y, 0)}{\partial t}=0
\end{array}\right.
$$

and over a square domain of $\Omega=\{(x, y) \mid-6 \leq x \leq 6$, $-6 \leq y \leq 6\}$. The numerical results of $u(x, y, t)$ are shown in Figure 2 for $t=1.0,2.0,3.0$, and 4.0, respectively. For better depicting the breakup of two orthogonal line solitons, we illustrate the contours of the superposition of the two orthogonal line solitons in Figure 3 that move separately from each other along the direction $y=-x$ over time. All numerical solutions are in good agreement with those available results in $[4,26,51]$.

In order to investigate the effects of the dissipative term on the behavior, the same numerical example is used with $\beta=0.5$ and 1.5 , respectively. Figures $3(\mathrm{~d}), 4(\mathrm{a})$, and $4(\mathrm{~b})$ illustrate the contours of the line solitons with varying damping factors when $t=4$, in which exist apparent propagation delay of the line solitons due to the presence of the dissipative term in the SG equation.

In order to quantitatively compare the numerical results with the literature, we adopt the energy for an undamped sine-Gordon equation $(\beta=0)$, which is conserved and defined as the following form [4]:

$$
E(t)=\frac{1}{2} \int_{\Omega}\left[u_{x}^{2}+u_{y}^{2}+u_{t}^{2}+2(1-\cos u)\right] \mathrm{d} \Omega
$$

The integration of the above equation over the problem domain $\Omega$ is calculated according to the trapezoidal rule. Table 1 exhibits the conservation of the energy $E(t)$ given by the present method, which agrees well with the literature $[4,26]$.

The convergence analysis of the present method is conducted based on the energy, $E(t)$, shown in Figure 5.

3.2. Example 2: Circular Ring Soliton (Damped) [4, 22]. For the numerical case, in which $\beta=0.05$ and $\phi(x, y)=1$, we numerically obtain the circular ring solitons with the specified initial conditions,

$$
\left\{\begin{array}{l}
u(x, y, 0)=4 \tan ^{-1} \exp \left(3-\sqrt{x^{2}+y^{2}}\right) \\
\frac{\partial u(x, y, 0)}{\partial t}=0
\end{array}\right.
$$

and in the problem domain of $\Omega=\{(x, y) \mid-7 \leq x$ $\leq 7,-7 \leq y \leq 7\}$. Figures 6 and 7 show the numerical solutions and their contours in term of $\sin (u(x, y, t) / 2)$ for $t=5.6$ and 8.4, respectively. Comparing the surfaces in Figure 6, a complete agreement can be observed with those published in [4] for the damped SG equation.

3.3. Perturbation of a Static Line Soliton [4, 52]. The perturbation of a line soliton is studied in the numerical example with $\beta=0.05$ and $\phi(x, y)=1$ and the specified initial conditions, 


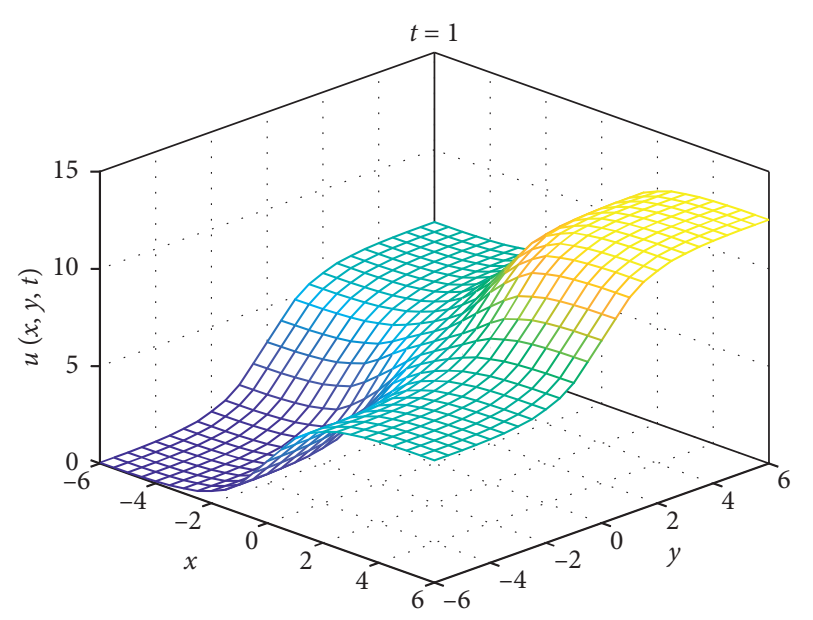

(a)

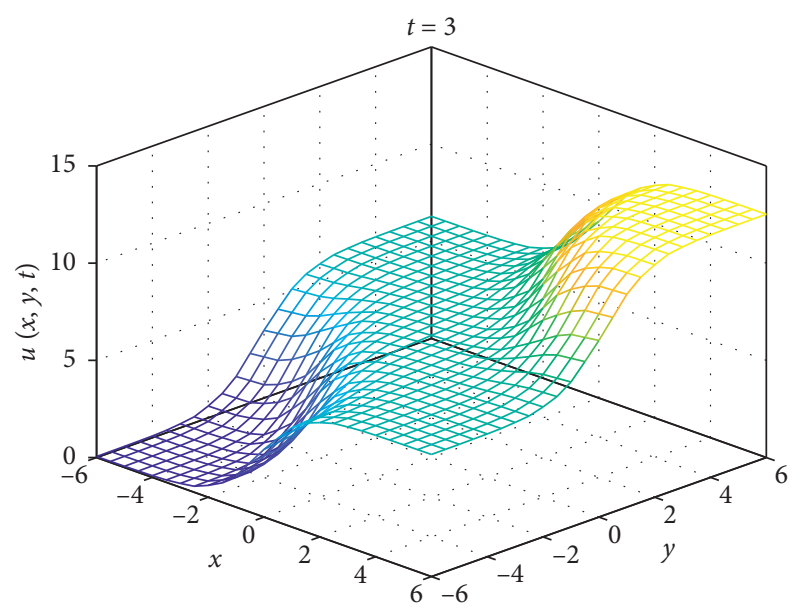

(c)

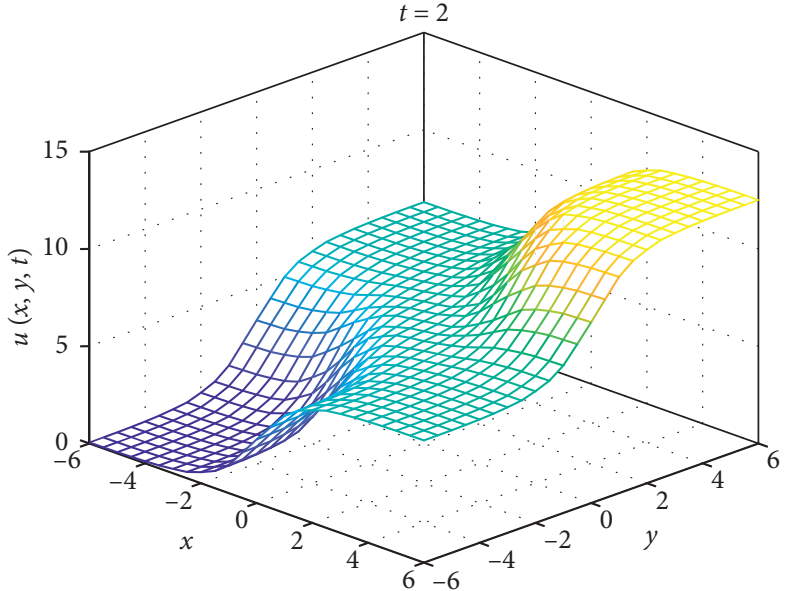

(b)

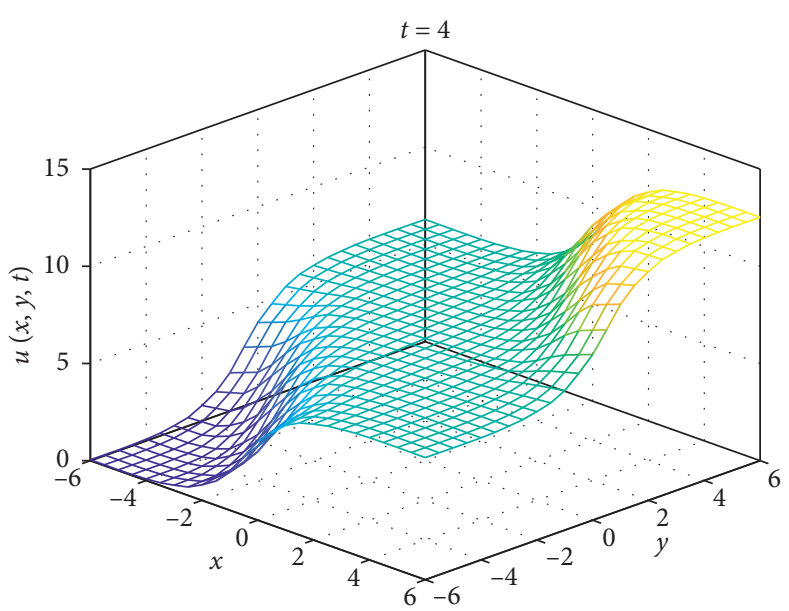

(d)

FIgURE 2: Superposition of two orthogonal line solitons $(\beta=0$ and $\phi(x, y)=1$ ). (a) $t=1.0$, (b) $t=2.0$, (c) $t=3.0$, and (d) $t=4.0$.

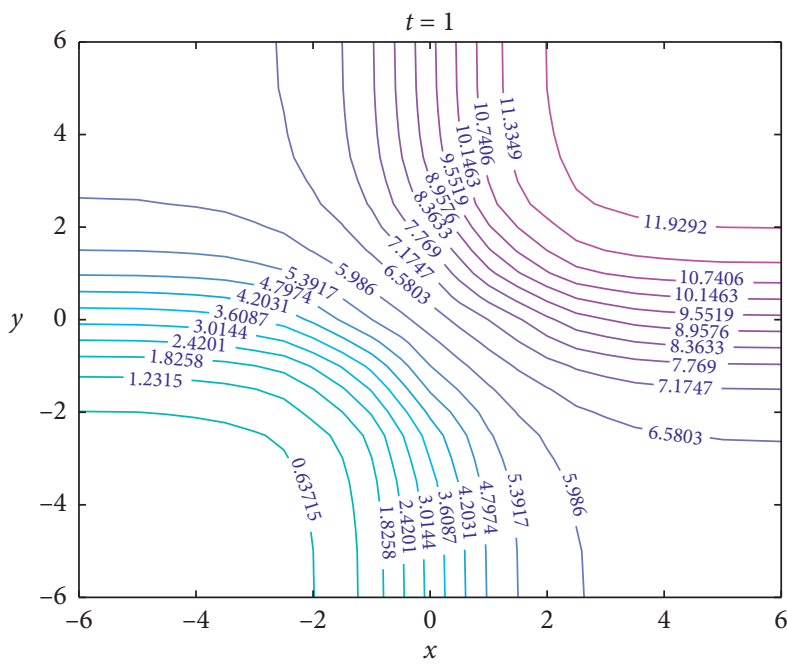

(a)

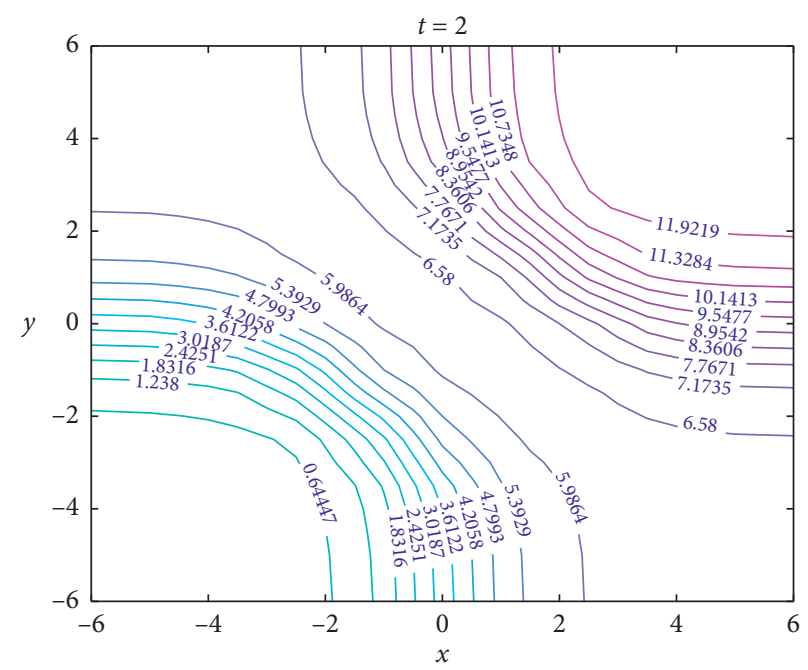

(b)

Figure 3: Continued. 


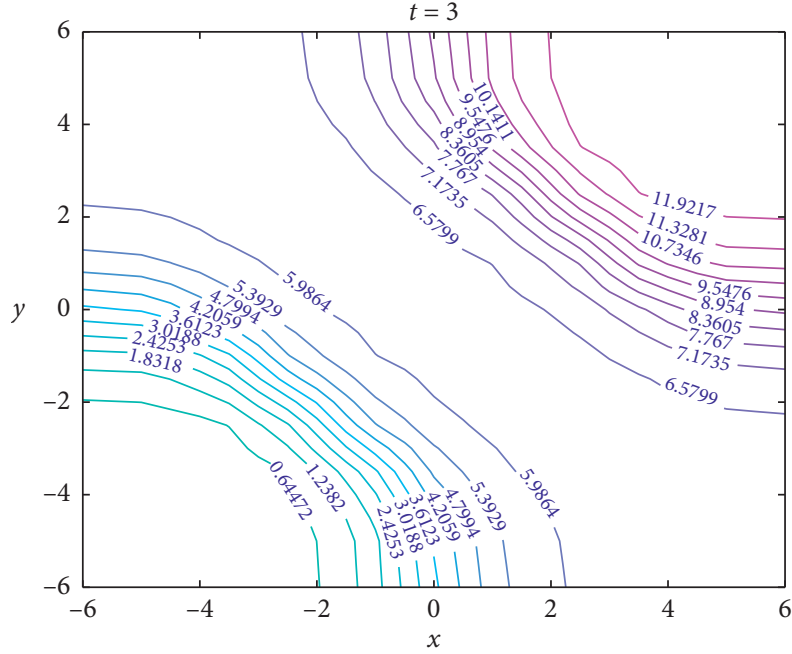

(c)

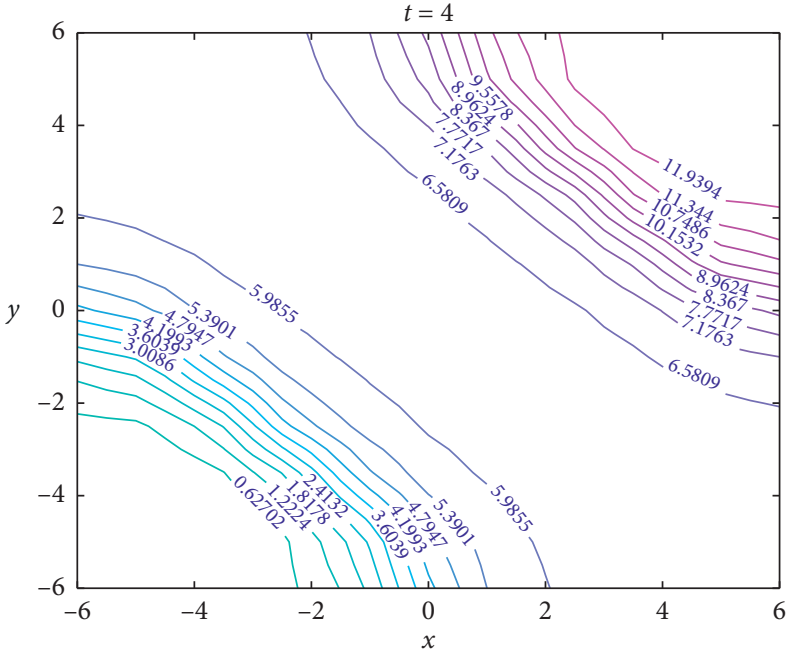

(d)

FIGURE 3: Contour for superposition of two orthogonal line solitons $(\beta=0$ and $\phi(x, y)=1)$. (a) $t=1.0$, (b) $t=2.0$, (c) $t=3.0$, and (d) $t=4$. .

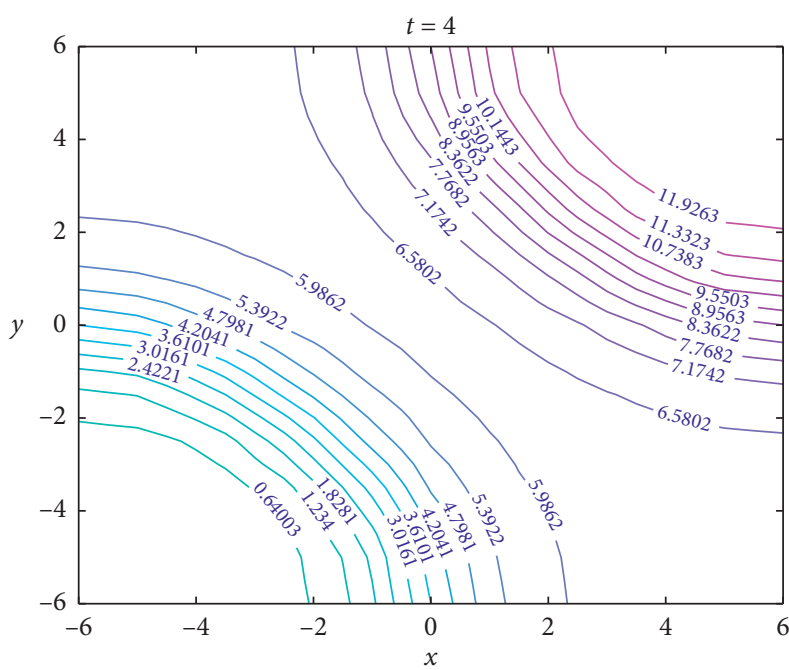

(a)

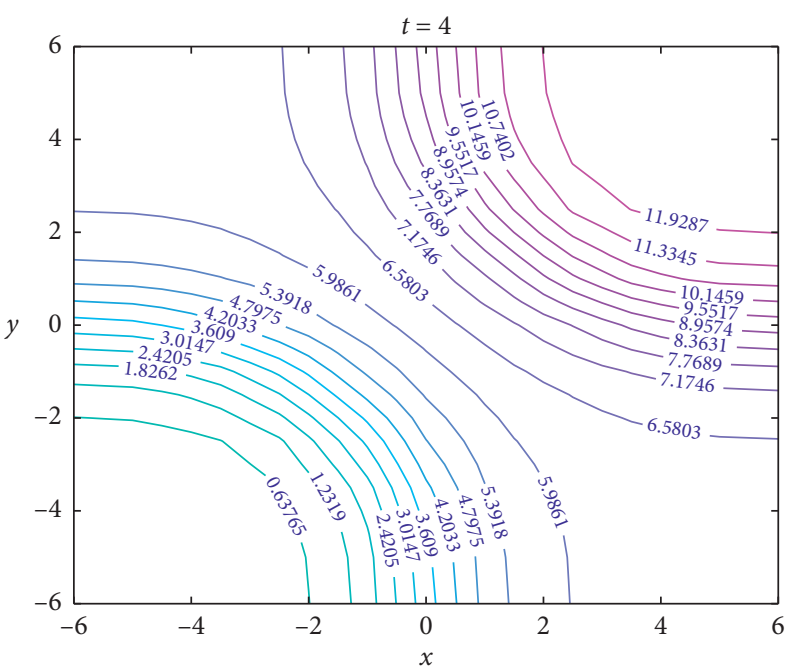

(b)

FIgURE 4: Contour for superposition of two orthogonal line solitons $(t=4.0, \phi(x, y)=1)$. (a) $\beta=0.5$ and (b) $\beta=1.5$.

TABLE 1: The energy $E(t)$ of superposition of two orthogonal line solitons $(\beta=0$ and $\phi(x, y)=1)$ for $t \in[0,7]$.

\begin{tabular}{lcccccccc}
\hline$t$ & $t=0$ & $t=1$ & $t=2$ & $t=3$ & $t=4$ & $t=5$ & $t=6$ & $t=7$ \\
\hline$E(t)[4]$ & - & 175.3027 & 175.3107 & 175.3289 & 175.3743 & 175.3921 & 175.4131 \\
$E(t)$ [26] & 175.5745 & 175.2927 & 175.2761 & 175.3188 & 175.3637 & 175.3899 & 175.4045 & 175.7865 \\
$E(t)$ (present) & 175.7503 & 175.7515 & 175.8155 & 175.9262 & 176.0511 & 176.2110 & 176.3888 & 176.5849 \\
\hline
\end{tabular}




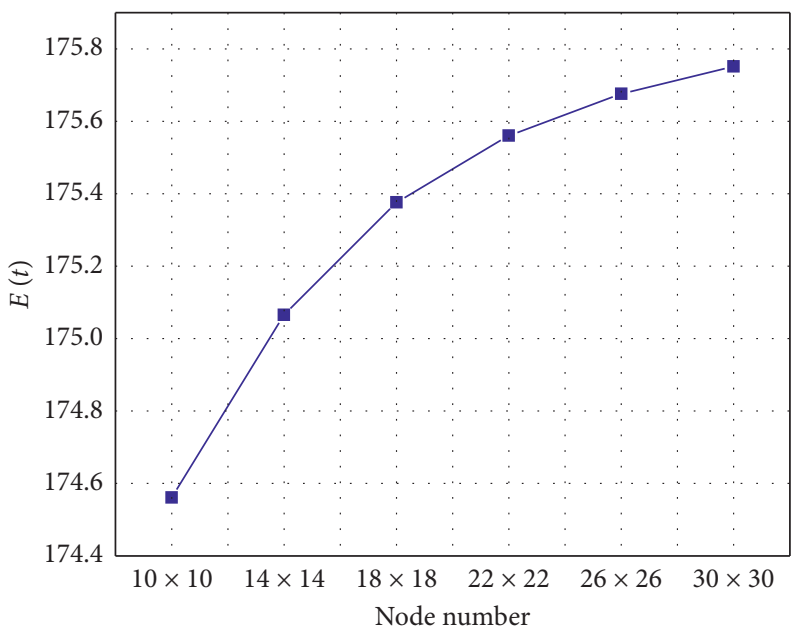

FIgURE 5: Relationship between the energy $E(t)$ and nodal distribution in Example 1.

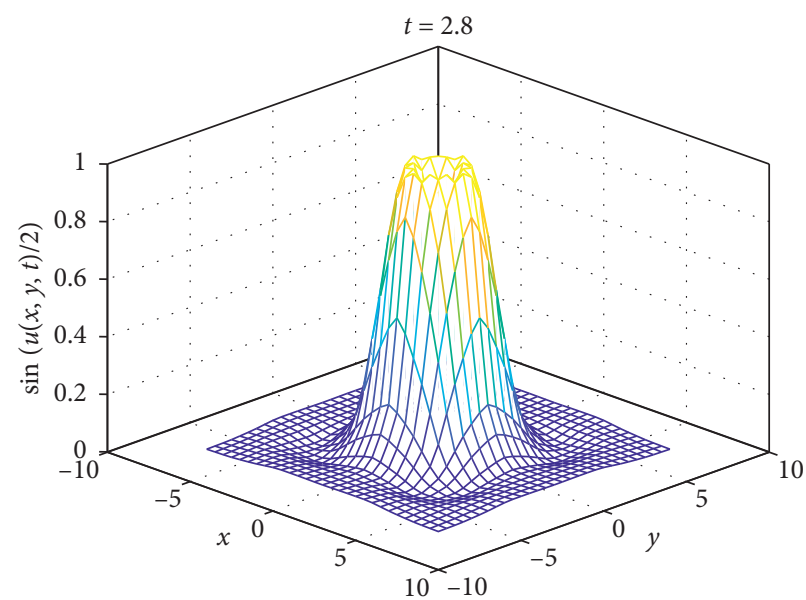

(a)

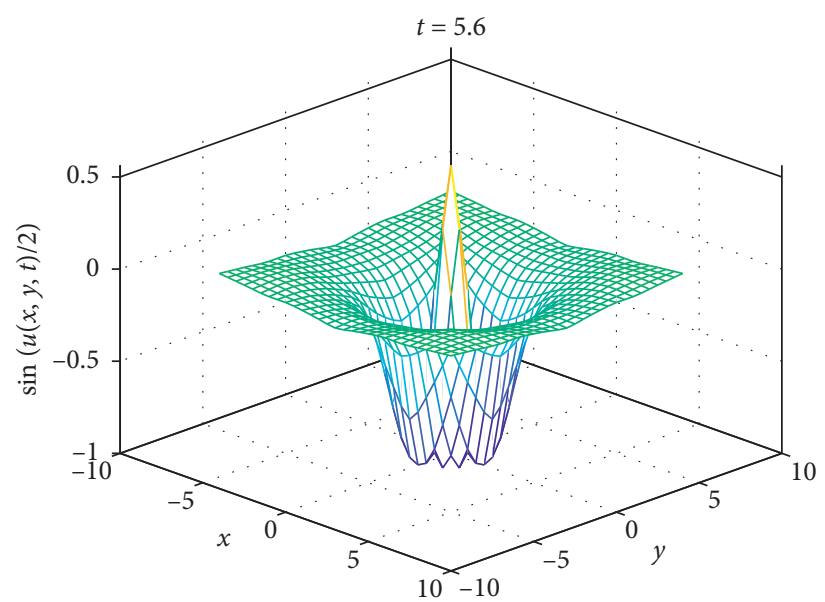

(b)

Figure 6: Circular ring solitons in terms of $\sin (u(x, y, t) / 2)(\beta=0.05$ and $\phi(x, y)=1)$. (a) $t=2.8$ and (b) $t=5.6$.

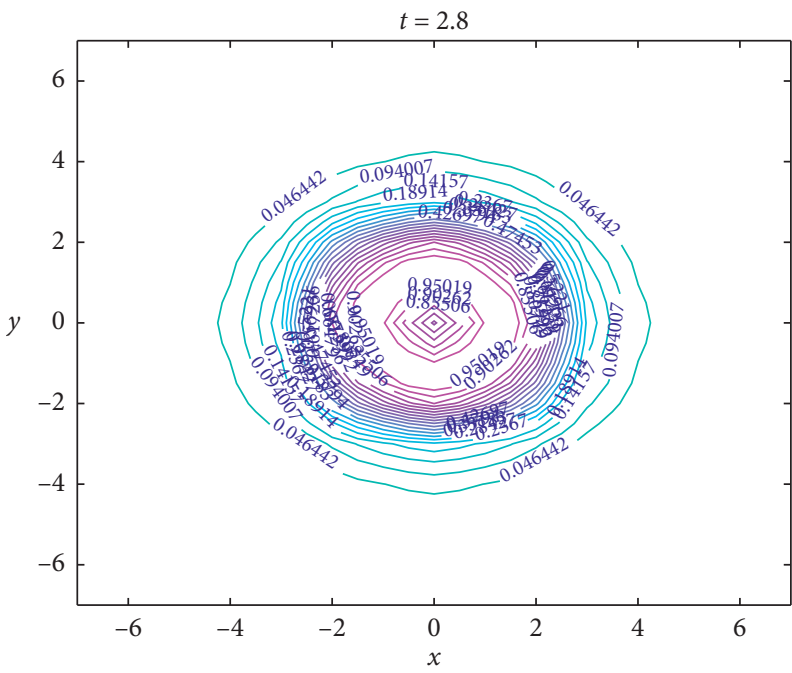

(a)

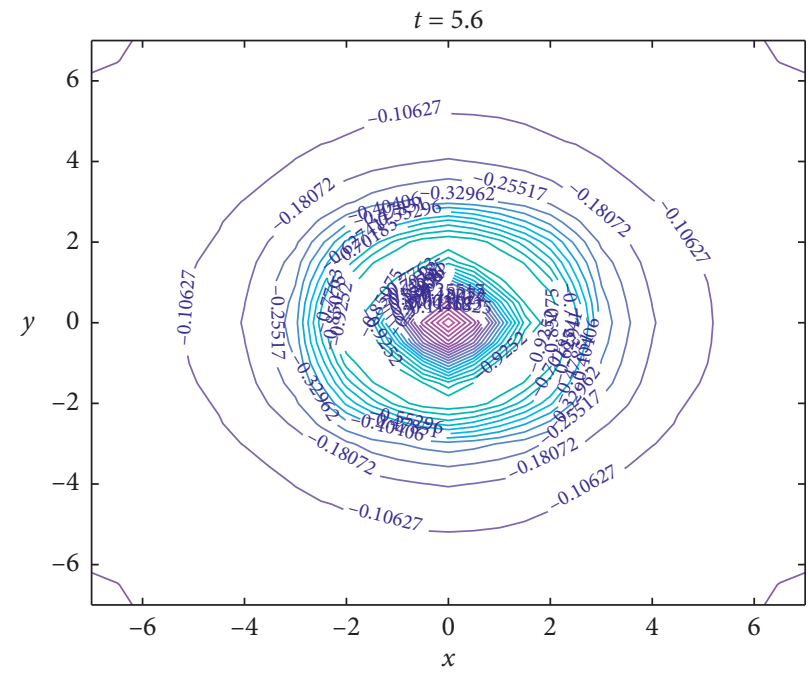

(b)

Figure 7: Contours of circular ring solitons in terms of $\sin (u / 2)(\beta=0.05$ and $\phi(x, y)=1)$. (a) $t=2.8$ and (b) $t=5.6$. 


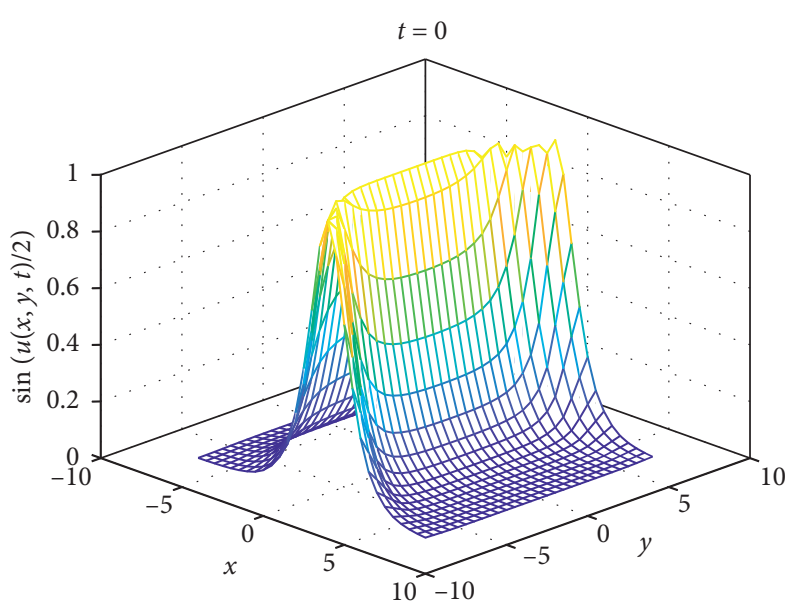

(a)

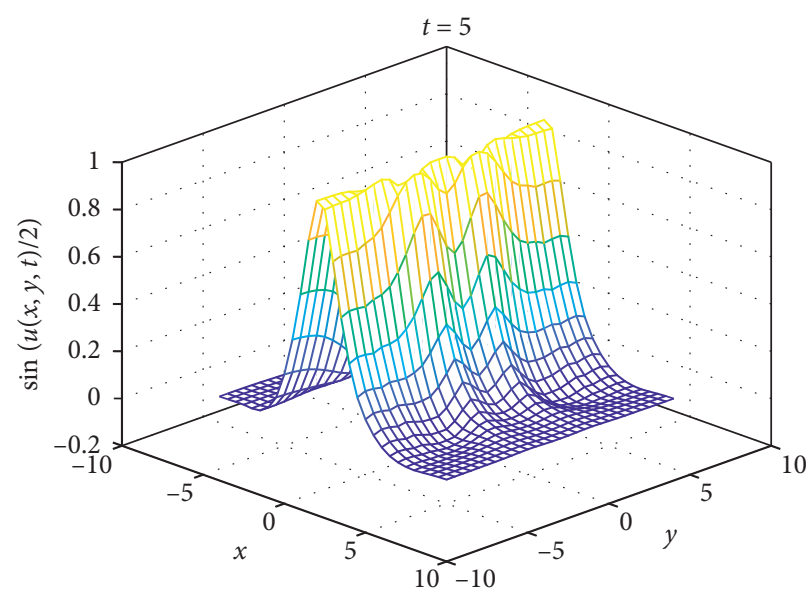

(c)

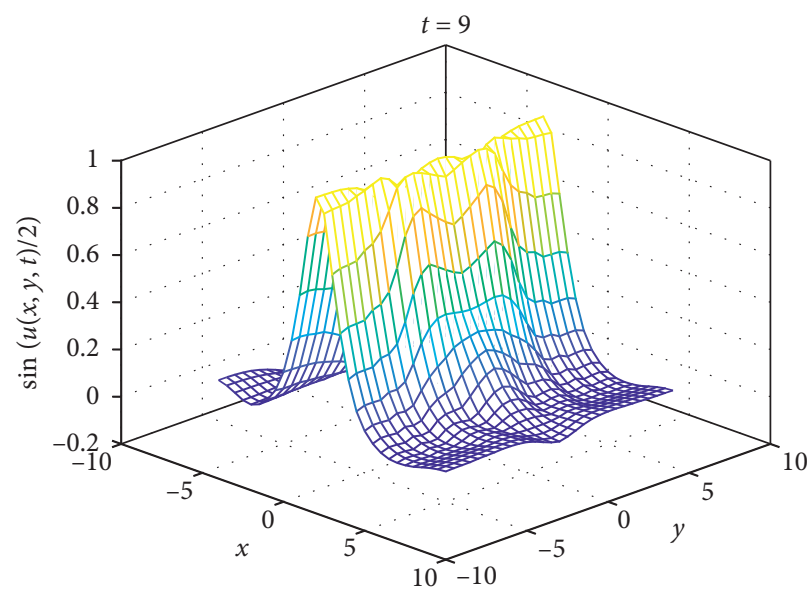

(e)

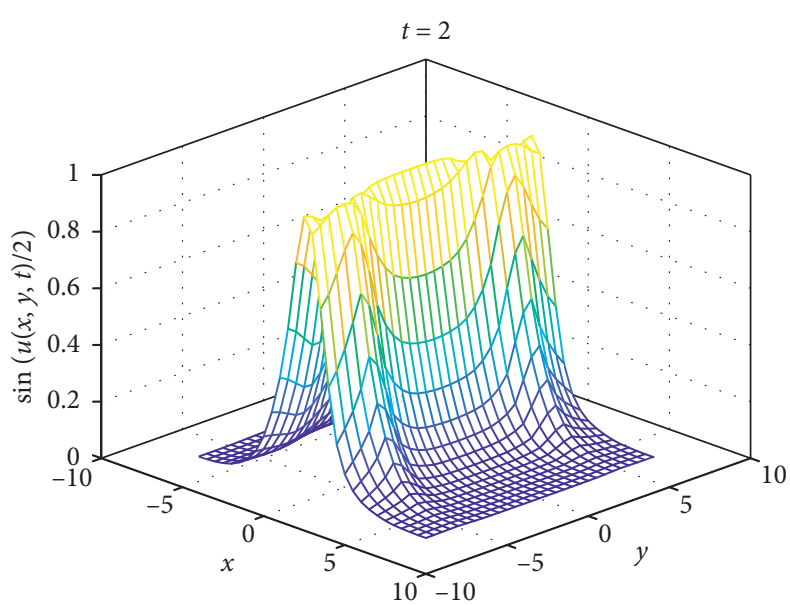

(b)

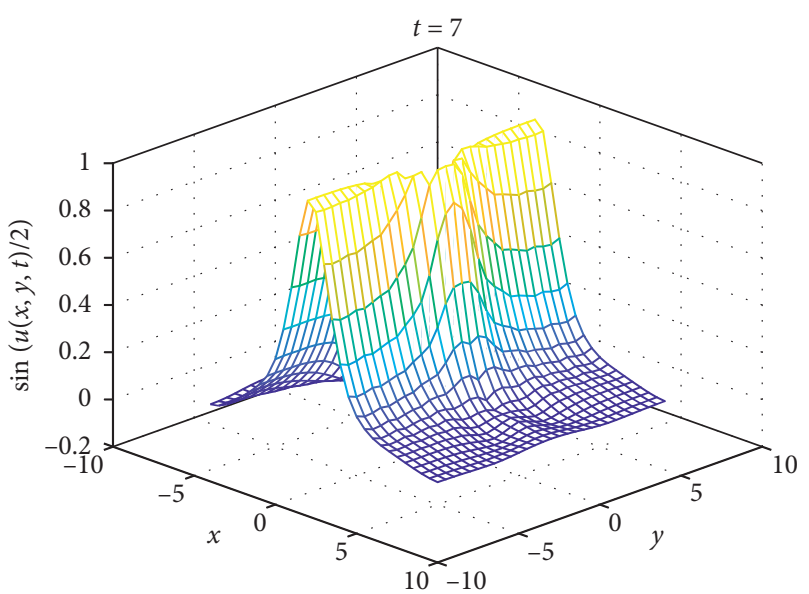

(d)

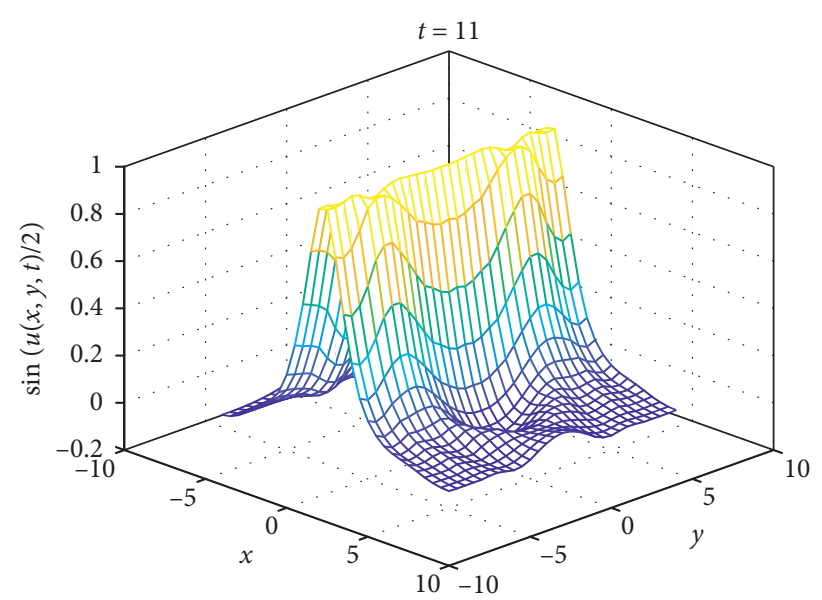

(f)

FiguRE 8: Symmetric perturbation of static line soliton in terms of $\sin (u(x, y, t) / 2)$ at different times $(\beta=0.05$ and $\phi(x, y)=1)$. (a) $t=0$, (b) $t=2.0$, (c) $t=5.0$, (d) $t=7.0$, (e) $t=9.0$, and (f) $t=11.0$. 


$$
\left\{\begin{array}{l}
u(x, y, 0)=4 \tan ^{-1} \exp (x+1-2 \operatorname{sech}(y+7)-2 \operatorname{sech}(y-7)), \\
\frac{\partial u(x, y, 0)}{\partial t}=0
\end{array}\right.
$$

over the problem domain $\Omega=\{(x, y) \mid-7 \leq x \leq 7,-7$ $\leq y \leq 7\}$. In Figure 8 which illustrates the symmetric perturbation of single line soliton in terms of $\sin (u(x, y, t) / 2)$ for $t=0,2,5,7,9$, and 11 , two symmetric dents firstly move towards each other, then collide at certain time, and proceed to move away from each other.

\section{Concluding Remarks}

In this study, the local Kriging meshless method has been extended to the 2+1-dimensional nonlinear sine-Gordon equation. The Kriging interpolation technique is used to approximate the two-dimensional field function, which leads to convenient implementation of imposing essential boundary conditions. A system of nonlinear discrete equations can be established based on the adoption of the local Petrov-Galerkin formulation and the center difference method for time discretization. The nonlinear algebraic equations are solved by applying the iterative technique and a predictor-corrector scheme. The numerical results are in good agreement with those available in the literature. The present meshless method is thus a potential alternative to other numerical methods, such as the finite element method and finite difference method, for dealing with a $2+1$-dimensional nonlinear sine-Gordon equation.

\section{Data Availability}

All results have been obtained by conducting the numerical procedure and the ideas can be shared for the researchers.

\section{Conflicts of Interest}

The authors declare that they have no conflicts of interest.

\section{Acknowledgments}

This work was supported by Hainan Provincial Natural Science Foundation of China (Grant no. 119MS039).

\section{References}

[1] R. K. Dodd, J. C. Eilbeck, J. D. Gibbon, and H. C. Morris, Solitons and Nonlinear Wave Equations, Academic, London, UK, 1982.

[2] A. Barone, F. Esposito, C. J. Magee, and A. C. Scott, "Theory and applications of the sine-Gordon equation," La Rivista del Nuovo Cimento, vol. 1, no. 2, pp. 227-267, 1971.

[3] A. M. Wazwaz, Partial Differential Equations and Solitary Waves Theory, Springer, Berlin, Germany, 2009.

[4] R. J. Cheng and K. M. Liew, "Analyzing two-dimensional sineGordon equation with the mesh-free reproducing kernel particle Ritz method," Computer Methods in Applied Mechanics and Engineering, vol. 245-246, pp. 132-143, 2012.
[5] S. Maitama and Y. F. Hamza, "An analytical method for solving nonlinear sine-Gordon equation," Sohag Journal of Mathematics, vol. 7, no. 1, pp. 5-10, 2020.

[6] L. Su, "Numerical solution of two-dimensional nonlinear sine-Gordon equation using localized method of approximate particular solutions," Engineering Analysis with Boundary Elements, vol. 108, pp. 95-107, 2019.

[7] M. Dehghan, M. Abbaszadeh, and A. Mohebbi, "An implicit RBF meshless approach for solving the time fractional nonlinear sine-Gordon and Klein-Gordon equations," Engineering Analysis with Boundary Elements, vol. 50, pp. 412-434, 2015.

[8] M. Dehghan and A. Ghesmati, "Numerical simulation of twodimensional sine-Gordon solitons via a local weak meshless technique based on the radial point interpolation method (RPIM)," Computer Physics Communications, vol. 181, no. 4, pp. 772-786, 2010.

[9] A.-M. Wazwaz, "The tanh method: exact solutions of the sineGordon and the sinh-Gordon equations," Applied Mathematics and Computation, vol. 167, no. 2, pp. 1196-1210, 2005.

[10] S. Johnson, P. Suarez, and A. Biswas, "New exact solutions for the sine-Gordon equation in $2+1$ dimensions," Computational Mathematics and Mathematical Physics, vol. 52, no. 1, pp. 98-104, 2012.

[11] W.-P. Zhong and M. Belić, "Special two-soliton solution of the generalized Sine-Gordon equation with a variable coefficient," Applied Mathematics Letters, vol. 38, pp. 122-128, 2014.

[12] M. R. Gao and H. T. Chen, "Hybrid solutions of three functions to the $(2+1)$-dimensional sine-Gordon equation," Acta Physica Sinica, vol. 61, no. 22, 2012.

[13] A. H. Salas, "Exact solutions of coupled sine-Gordon equations," Nonlinear Analysis: Real World Applications, vol. 11, no. 5, pp. 3930-3935, 2010.

[14] T. Aktosun, F. Demontis, and C. van der Mee, "Exact solutions to the sine-Gordon equation," Journal of Mathematical Physics, vol. 51, no. 12, p. 123521, 2010.

[15] W.-X. Chen and J. Lin, "Some new exact solutions of (1+2)dimensional sine-Gordon equation," Abstract and Applied Analysis, vol. 2014, Article ID 645456, 8 pages, 2014.

[16] M. Dehghan and A. Shokri, "A numerical method for onedimensional nonlinear sine-Gordon equation using collocation and radial basis functions," Numerical Methods for Partial Differential Equations, vol. 24, no. 2, pp. 687-698, 2008.

[17] A. G. Bratsos and E. H. Twizell, "A family of parametric finitedifference methods for the solution of the sine-Gordon equation," Applied Mathematics and Computation, vol. 93, no. 2-3, pp. 117-137, 1998.

[18] M. Dehghan and D. Mirzaei, "The boundary integral equation approach for numerical solution of the one-dimensional sineGordon equation," Numerical Methods for Partial Differential Equations, vol. 24, no. 6, pp. 1405-1415, 2008.

[19] M. S. H. Chowdhury and I. Hashim, "Application of homotopy-perturbation method to Klein-Gordon and sineGordon equations," Chaos, Solitons \& Fractals, vol. 39, no. 4, pp. 1928-1935, 2009.

[20] S. S. Ray, "A numerical solution of the coupled sine-Gordon equation using the modified decomposition method," Applied Mathematics and Computation, vol. 175, no. 2, pp. 1046-1054, 2006.

[21] J. Chen, Z. Chen, and S. Cheng, "Multilevel augmentation methods for solving the sine-Gordon equation," Journal of Mathematical Analysis and Applications, vol. 375, no. 2, pp. 706-724, 2011. 
[22] J. Argyris, M. Haase, and J. C. Heinrich, "Finite element approximation to two-dimensional sine-Gordon solitons," Computer Methods in Applied Mechanics and Engineering, vol. 86, no. 1, pp. 1-26, 1991.

[23] K. Djidjeli, W. G. Price, and E. H. Twizell, "Numerical solutions of a damped Sine-Gordon equation in two space variables," Journal of Engineering Mathematics, vol. 29, no. 4, pp. 347-369, 1995.

[24] Q. Sheng, A. Q. M. Khaliq, and D. A. Voss, "Numerical simulation of two-dimensional sine-Gordon solitons via a split cosine scheme," Mathematics and Computers in Simulation, vol. 68, no. 4, pp. 355-373, 2005.

[25] A. G. Bratsos, "The solution of the two-dimensional sineGordon equation using the method of lines," Journal of Computational and Applied Mathematics, vol. 206, no. 1, pp. 251-277, 2007.

[26] D. Mirzaei and M. Dehghan, "Meshless local Petrov-Galerkin (MLPG) approximation to the two dimensional sine-Gordon equation," Journal of Computational and Applied Mathematics, vol. 233, no. 10, pp. 2737-2754, 2010.

[27] X. Li, "Meshless numerical analysis of a class of nonlinear generalized Klein-Gordon equations with a well-posed moving least squares approximation," Applied Mathematical Modelling, vol. 48, pp. 153-182, 2017.

[28] D. E. Beskos, "Boundary element methods in dynamic analysis," Applied Mechanics Reviews, vol. 40, no. 1, pp. 1-23, 1987.

[29] R. Huang, S. Zheng, Z. Liu, and T. Y. Ng, "Recent advances of the constitutive models of smart materials-hydrogels and shape memory polymers," International Journal of Applied Mechanics, vol. 12, no. 02, p. 2050014, 2020.

[30] T. Belytschko, Y. Y. Lu, and L. Gu, "Element-free Galerkin methods," International Journal for Numerical Methods in Engineering, vol. 37, no. 2, pp. 229-256, 1994.

[31] J. Cheng, "Data analysis of the factors influencing the industrial land leasing in Shanghai based on mathematical models," Mathematical Problems in Engineering, vol. 2020, Article ID 9346863, 2020.

[32] J. Cheng, "Analyzing the factors influencing the choice of the government on leasing different types of land uses: evidence from Shanghai of China," Land Use Policy, vol. 90, p. 104303, 2020.

[33] Y. M. Cheng and M. J. Chen, "A boundary element-free method for linear elasticity," Acta Mechanica Sinica, vol. 35, no. 2, pp. 181-186, 2003.

[34] B. Wang, Y. Ma, and Y. Cheng, "The improved complex variable element-free Galerkin method for bending problem of thin plate on elastic foundations," International Journal of Applied Mechanics, vol. 11, no. 10, p. 1950105, 2019.

[35] F. Liu and Y. Cheng, "The improved element-free Galerkin method based on the nonsingular weight functions for inhomogeneous swelling of polymer gels," International Journal of Applied Mechanics, vol. 10, no. 4, p. 1850047, 2018.

[36] Q. Wu, P. P. Peng, and Y. M. Cheng, "The interpolating element-free Galerkin method for elastic large deformation problems," Science China Technological Sciences, vol. 63, 2020.

[37] Q. Wu, F. B. Liu, and Y. M. Cheng, "The interpolating element-free Galerkin method for three-dimensional elastoplasticity problems," Engineering Analysis with Boundary Elements, vol. 115, pp. 156-167, 2020.

[38] D. Liu and Y. M. Cheng, "The interpolating element-free Galerkin (IEFG) method for three-dimensional potential problems," Engineering Analysis with Boundary Elements, vol. 108, pp. 115-123, 2019.
[39] Z. J. Meng, H. Cheng, L. D. Ma, and Y. M. Cheng, "The dimension splitting element-free Galerkin method for 3D transient heat conduction problems," Science China Physics, Mechanics \& Astronomy, vol. 62, no. 4, Article ID 40711, 2019.

[40] L. Ma, Z. Meng, J. Chai, and Y. Cheng, "Analyzing 3D advection-diffusion problems by using the dimension splitting element-free Galerkin method," Engineering Analysis with Boundary Elements, vol. 111, pp. 167-177, 2020.

[41] Z. J. Meng, H. Cheng, L. D. Ma, and Y. M. Cheng, "The dimension split element-free Galerkin method for three-dimensional potential problems," Acta Mechanica Sinica, vol. 34, no. 3, pp. 462-474, 2018.

[42] H. Cheng, M. Peng, Y. Cheng, and Z. Meng, "The hybrid complex variable element-free Galerkin method for 3D elasticity problems," Engineering Structures, vol. 219, p. $110835,2020$.

[43] P. P. Peng, Q. Wu, and Y. M. Cheng, "The dimension splitting reproducing kernel particle method for three-dimensional potential problems," International Journal for Numerical Methods in Engineering, vol. 121, no. 1, pp. 146-164, 2020.

[44] P. Zhu, L. W. Zhang, and K. M. Liew, "Geometrically nonlinear thermomechanical analysis of moderately thick functionally graded plates using a local Petrov-Galerkin approach with moving Kriging interpolation," Composite Structures, vol. 107, pp. 298-314, 2014.

[45] P. Zhu and K. M. Liew, "A local Kriging meshless method for free vibration analysis of functionally graded circular plates in thermal environments," Procedia Engineering, vol. 31, pp. 1089-1094, 2012.

[46] P. Zhu and K. M. Liew, "Free vibration analysis of moderately thick functionally graded plates by local Kriging meshless method," Composite Structures, vol. 93, no. 11, pp. 2925-2944, 2011.

[47] L. W. Zhang, P. Zhu, and K. M. Liew, "Thermal buckling of functionally graded plates using a local Kriging meshless method," Composite Structures, vol. 108, pp. 472-492, 2014.

[48] R. Olea, Geostatistics for Engineers and Earth Scientists, Kluwer Academic, Boston, MA, USA, 1999.

[49] Z. Liu, G. Wei, and Z. Wang, "The radial basis reproducing kernel particle method for geometrically nonlinear problem of functionally graded materials," Applied Mathematical Modelling, vol. 85, pp. 244-272, 2020.

[50] Z. Liu, G. Wei, Z. Wang, and J. Qiao, "The meshfree analysis of geometrically nonlinear problem based on radial basis reproducing kernel particle method," International Journal of Applied Mechanics, vol. 12, no. 4, p. 2050044, 2020.

[51] P. L. Christiansen and P. S. Lomdahl, "Numerical study of $2+1$ dimensional Sine-Gordon solitons," Physica D: Nonlinear Phenomena, vol. 2, no. 3, pp. 482-494, 1981.

[52] A. G. Bratsos, "An explicit numerical scheme for the SineGordon equation in $2+1$ dimensions," Applied Numerical Analysis \& Computational Mathematics, vol. 2, no. 2, pp. 189-211, 2005. 